\title{
Multi-wavelength selection and identification of gamma-ray blazar candidates
}

\author{
Alessandro Paggi ${ }^{1}$, R. D’Abrusco ${ }^{1}$, F. Massaro ${ }^{2}$, M. Landoni ${ }^{1,3}$, \\ D. Milisavljevic ${ }^{1}$, N. Masetti ${ }^{4}$, F. Ricci ${ }^{5}$, E. Jiménez-Bailón ${ }^{6}$, \\ V. Chavushyan ${ }^{7}$, M. Giroletti ${ }^{4}$, H. A. Smith ${ }^{1}$, R. Margutti ${ }^{1}$, \\ G. Tosti ${ }^{8}$, J. R. Martinéz-Galarza ${ }^{1}$, H. Otí-Floranes ${ }^{6}$, J. E. Grindlay ${ }^{1}$, \\ S. Funk ${ }^{2}$, C. C. Cheung ${ }^{9}$, L. Chomiuk ${ }^{10}$ and J. Strader ${ }^{10}$ \\ ${ }^{1}$ Harvard-Smithsonian Center for Astrophysics \\ 60 Garden Street, Cambridge, MA 02138, USA \\ email: apaggi@cfa.harvard.edu \\ ${ }^{2}$ SLAC - National Laboratory and Kavli Institute for Particle Astrophysics and Cosmology \\ 2575 Sand Hill Road, Menlo Park, CA 94025, USA \\ ${ }^{3}$ INAF - Osservatorio Astronomico di Brera \\ Via Emilio Bianchi 46, I-23807 Merate, Italy \\ ${ }^{4}$ INAF - Istituto di Astrosica Spaziale e Fisica Cosmica di Bologna \\ via Gobetti 101, 40129, Bologna, Italy \\ ${ }^{5}$ Dipartimento di Matematica e Fisica, Universit Roma Tre \\ via della Vasca Navale 84, I-00146, Roma, Italy \\ ${ }^{6}$ Instituto de Astronomía, Universidad Nacional Autónoma de México \\ Apdo. Postal 877, Ensenada, 22800 Baja California, México \\ ${ }^{7}$ Instituto Nacional de Astrofísica, Óptica y Electrónica \\ Apartado Postal 51-216, 72000 Puebla, México \\ ${ }^{8}$ Dipartimento di Fisica, Università degli Studi di Perugia \\ 06123 Perugia, Italy \\ ${ }^{9}$ Space Science Division, Naval Research Laboratory \\ Washington, DC 20375-5352, USA \\ ${ }^{10}$ Department of Physics and Astronomy, Michigan State University \\ East Lansing, MI 48824, USA
}

\begin{abstract}
A significant fraction $(\sim 30 \%)$ of the gamma-ray sources detected by the Fermi Gamma-ray Space Telescope is still of unknown origin, being not yet associated with counterparts at lower energies. Many unidentified gamma-ray sources (UGSs) could be blazars, the largest identified population of extragalactic gamma-ray sources and the rarest class of active galactic nuclei. In particular, it has been found that blazars occupy a defined region in WISE three dimensional color space, well separated from that occupied by other sources in which thermal emission prevails. For farther sources with weaker IR emission, additional informations can be obtained combining WISE data with X-ray or radio emission. Alternatively, the lowfrequency radio emission can be used for identifying potential gamma-ray candidate blazars. However, optical spectroscopic observations represent the tell-tale tool to confirm the exact nature of these sources. To this end, an extensive observational campaign has been performed with several optical telescopes, aimed at pinpointing the exact nature of gamma-ray candidate blazars selected with the different selection methods mentioned above. The results of this campaign lead to the discovery of 60 new gamma-ray blazars, thus confirming the effectiveness of these selection criteria.
\end{abstract}

Keywords. galaxies: active, BL Lacertae objects: general, galaxies: jets, radio continuum: galaxies, infrared: galaxies, X-rays: galaxies, gamma rays: observations 


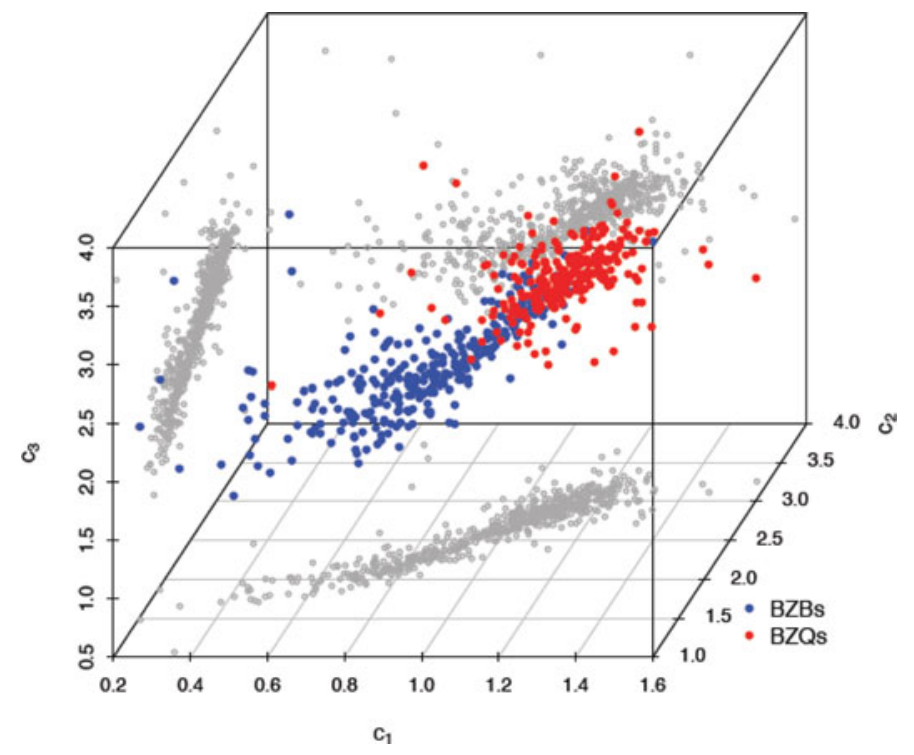

Figure 1. Scatterplot of the WFB sources in the three-dimensional WISE color space. The spectral class of the WFB sources is color-coded, while the three distributions of gray points represent the projections of the WFB sample in the three-dimensional color space onto the three two-dimensional color-color planes generated by the WISE colors $\mathrm{c} 1=[3.4]-[4.6], \mathrm{c} 2=[4.6]-[12]$ and $\mathrm{c} 3=[12]-[22]$, respectively.

\section{Introduction}

About $1 / 3$ of the $\gamma$-ray sources listed in the 2 nd Fermi-LAT catalog (2FGL, Nolan et al. 2012) have not yet been associated with counterparts at lower energies. A precise knowledge of the number of unidentified gamma-ray sources (UGSs) is extremely relevant since for example it could help to provide the tightest constraint on the dark matter models ever determined (Berlin \& Hooper 2014). Many UGSs could be blazars, the largest identified population of extragalactic $\gamma$-ray sources, but how many are actually blazars is not yet known due in part to the incompleteness of the catalogs used for the associations (Ackermann et al. 2011). The first step to reduce the number of UGSs is therefore to recognize those that could be blazars.

Blazars are the rarest class of active galactic nuclei, dominated by variable, non-thermal radiation over the entire electromagnetic spectrum (e.g., Urry \& Padovani 1995; Giommi, Padovani \& Polenta 2013). Their observational properties are generally interpreted in terms of a relativistic jet aligned within a small angle to our line of sight (Blandford \& Rees 1978).

Blazars have been classified as BL Lacs and FSRQs (or BZBs and BZQs according to the nomenclature proposed by Massaro et al. 2013), with the latter showing similar optical spectra except for the stronger emission lines, as well as higher radio polarization. In particular, if the only spectral features observed are emission lines with rest frame equivalent width $E W \leqslant 5 \AA$ the object is classified as a BZB (Sickel et al. 1991; Stocke \& Rector 1997), otherwise it is classified as BZQ (Lurent-Muehleisen et al. 1999; Massaro et al. 2013). Systematic projects aimed at obtaining optical spectroscopic observations of blazars are currently carried out by different groups (see, e.g., Sbaruffati et al. 2006; Sbaruffati et al. 2009; Landoni et al. 2012; Landoni et al. 2013; Shaw et al. 2013a).

Here we present the main results of an extensive campaign of optical spectroscopic observations carried on during 2013 and 2014 with different facilities (both in the northern 

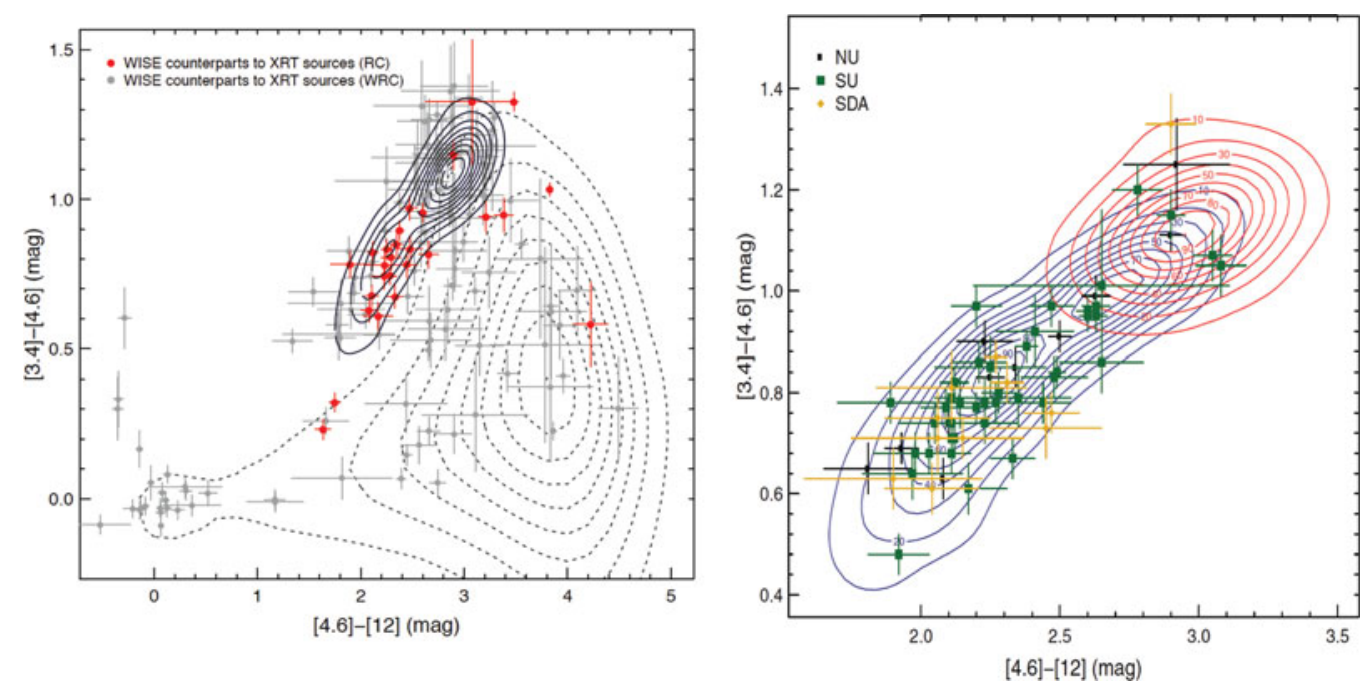

Figure 2. (Left panel) Projection of the three-dimensional WISE color space on the two-dimensional[3.4]-[4.6] [4.6]-[12] color-color plane for Swift-XRT sources with a WISE counterpart. Black lines represent the two-dimensional densities of WISE counterparts to know $\gamma$-ray blazars evaluated using the KDE technique, with the outermost line indicating the $90 \%$ density contour normalized to the peak density. Grey circles represent XRT-PC sources without a radio counterpart (WRC), and red circles represent the XRT-PC sources with a radio counterpart (RC). Black dashed lines represent isodensity contours of generic WISE sources. (Right panel) Isodensity contours generated by the $\mathrm{KDE}$ technique in the [3.4]-[4.6] [12] color color diagram for the BZBs (blue) and the BZQs (red). Points overlaid to the contours show the location of the selected radio candidates with IR colors consistent with the $\gamma$-ray blazar population for the sources in the three different samples analyzed: NU (NVSS survey, black circles), SU (SUMSS survey, green squares), and SDA (ATCA survey, yellow diamonds).

and southern hemisphere) aimed at pinpointing the exact nature of gamma-ray candidate blazars selected with different methods described in Sect. 2. The results of this campaign lead to the discovery of 60 new gamma-ray blazars, thus confirming the effectiveness of these selection criteria.

\section{Selection methods}

Recently, D'Abrusco et al. (2013) proposed an association procedure to recognize $\gamma$ ray blazar candidates on the basis of their positions in the three-dimensional WISE color space. As a matter of fact, blazars - whose emission is dominated by beamed, non thermal emission - occupy a defined region in such a space (the WISE Fermi Blazar locus, WFB, see Fig. 1), well separated from that occupied by other sources in which thermal emission prevails (Massaro et al. 2011; D'Abrusco et al. 2012). Applying this method, Cowperthwaite et al. (2013) recently identified thirteen gamma-ray emitting blazar candidates from a sample of 102 previously unidentified sources selected from Astronomer's Telegrams and the literature.

Massaro et al. (2013a) applied the classification method proposed by D'Abrusco et al. (2013) to 258 UGSs and 210 active galaxies of uncertain type (AGUs) listed in the 2FGL finding candidate blazar counterparts for 141 UGSs and 125 AGUs. The classification method proposed by D'Abrusco et al. (2013), however, can only be applied to sources detected in all 4 WISE bands, i.e., 3.4, 4.6, 12 and $22 \mu \mathrm{m}$. 

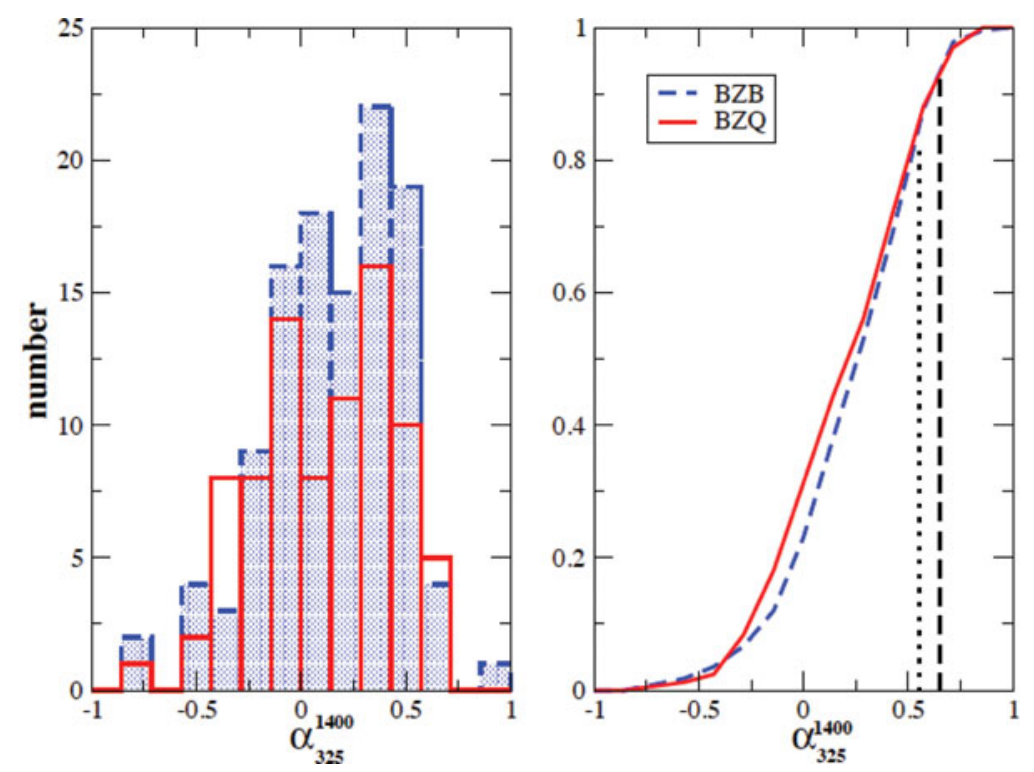

Figure 3. The distributions of the radio spectral index between 325 and $1400 \mathrm{GHz}\left(\alpha_{325}^{1400}\right)$ of all the identified blazars in the low radio frequency Gamma-ray Blazar sample sample, BZBs (blue) and BZQs (red). The two vertical lines mark the 0.55 (dotted) and 0.65 (dashed) values of $\alpha_{325}^{1400}$.

Using the X-ray emission in place of the $22 \mu \mathrm{m}$ detection, Paggi et al. (2013) proposed a method to select $\gamma$-ray blazar candidates among Swift-XRT sources considering those that feature a WISE counterpart detected at least in the first 3 bands, and with IR colors compatible with the $90 \%$ two-dimensional densities of known $\gamma$-ray blazar evaluated using the Kernel Density Estimation (KDE) technique (Richards et al. 2004; D'Abrusco, Longo \& Walton 2009; Laurino et al. 2011 and reference therein), so selecting 37 new $\gamma$-ray blazar candidates (Fig. 2, left panel). Similarly, using the radio emission as additional information, Massaro et al. (2013b) investigated all the radio sources in NVSS and SUMSS surveys that lie within positional uncertainty region of Fermi UGSs and, considering those sources with IR colors compatible with the $90 \%$ two-dimensional KDE densities of known $\gamma$-ray blazar, selected 66 additional $\gamma$-ray blazar candidates (Fig. 2, right panel).

Finally, Massaro et al. (2013c) investigated the low-frequency radio emission of blazars and searched for sources with similar features combining the information derived from the WENSS and NVSS surveys, identifying $26 \gamma$-ray candidate blazars in the Fermi LAT the positional uncertainty region of 21 UGSs.

\section{Observations and Results}

During 2013 and 2014 we carried on an extensive campaign of optical spectroscopic observations of candidate $\gamma$-ray blazar candidates selected with the methods described in Sect. 2, mainly thanks to a joint Fermi-NOAO proposal approved in 2013 (PI: D'Abrusco), making use of MMT, OAN, SOAR and KPNO telescopes. The results of these campaign are presented in several published and submitted papers (Paggi et al. 2014; Landoni et al. 2014; Massaro et al. 2014; Ricci et al. 2014), and here we recap the main points.

So far we observed $60 \gamma$-ray blazar candidates, and all of them show featureless BZB optical spectra or QSO-like spectra typical of BZQs, thus confirming the reliability of our 

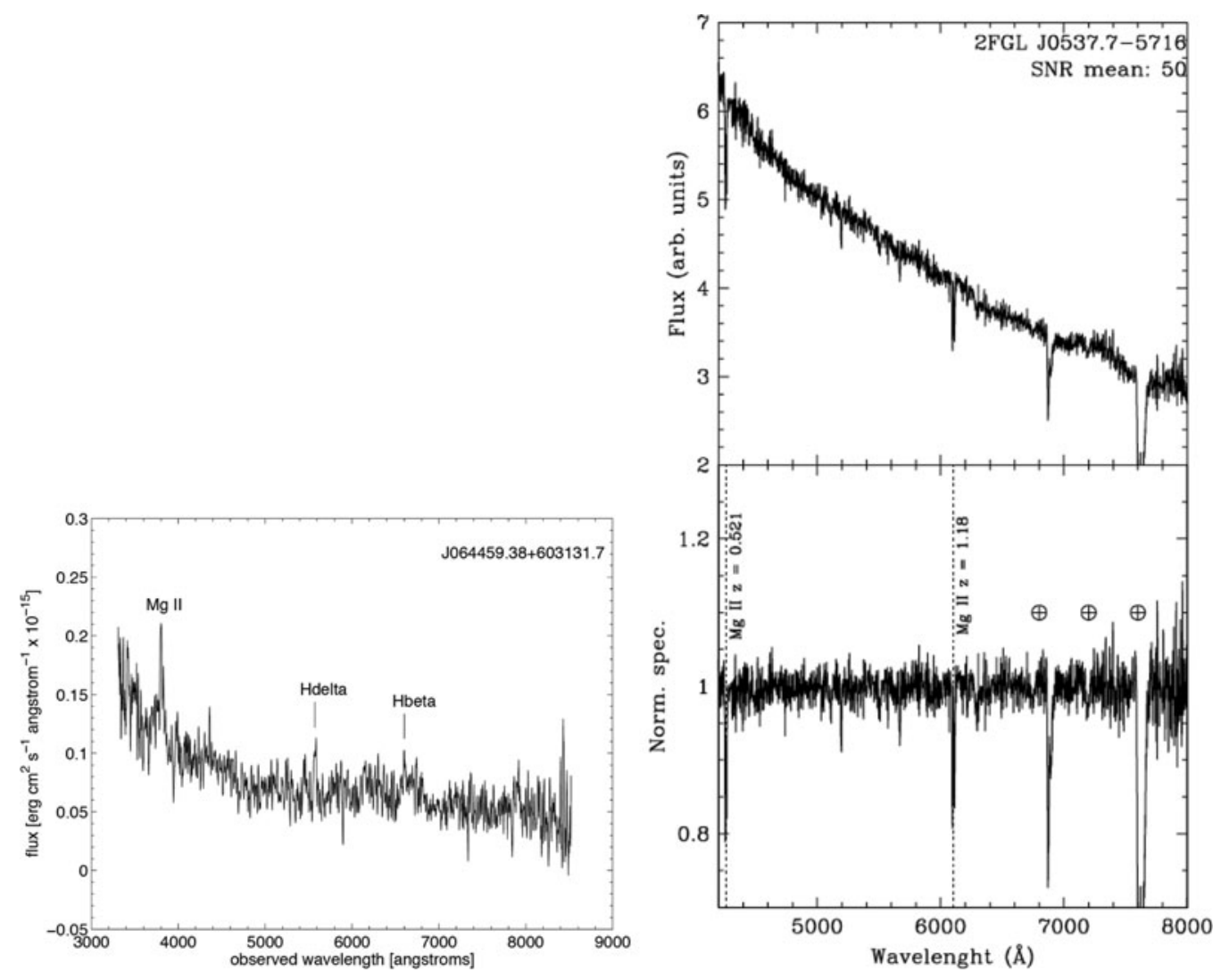

Figure 4. (Left panel). Optical spectra of the WISE $\gamma$-ray blazar candidate WISE J064459.38+603131.7 associated with Fermi-LAT UGS 2FGL J0644.6+6034 obtained with MMT Blue Channel Spectrograph. (Right panel). 2FGL J0537.7-5716 optical spectrum obtained at SOAR with Goodman High Throughput Spectrograph. Top: Flux calibrated (relative units) spectrum of the source. Bottom: Normalised spectrum.

different approaches in selecting $\gamma$-ray candidate blazars. In particular, the spectrum of WISEJ064459.38+603131.7 (Fig. 4, left panel), is somewhat reminiscent of weak emission line quasar spectra Shemmer et al. (2006), Shemmer et al. (2009), but the blazar identification for this source appears problematic. In fact the WISEJ064459.38+603131.7 is not detected by NVSS survey, so we can only put an upper limit on its flux $\sim 2.5 \mathrm{mJy}$. Even if it is possible that deeper radio observations will detect emission from the source, blazars are traditionally defined as radio-loud sources basing on present radio data. All confirmed blazar from BZCAT are in fact detected at $1.4 \mathrm{GHz}$ with fluxes $\gtrsim 1 \mathrm{mJy}$, and radio-quiet blazars are extremely rare objects Londish et al. (2004). We have been also able to evaluate redshifts for a number of our sources. In particular, we highlight the lower limit redshift that we put for the BZB source 2FGL J0537.7-5716 where multiple $\mathrm{Mg}$ II absorption system are detected. The first one is found to be at redshift $z=0.521$ while the second one is at $z=1.18$ making this source the third the most distant BZB ever observed.

In conclusion the results of our observing campaign seem confirm the reliability of our multi-wavelength methods in selecting selecting $\gamma$-ray candidate blazars. There could be possible contamination by different source classes in these selection procedures (e.g., Stern \& Assef 2013) and such degeneracy can be only removed with optical spectroscopic 
observations (Masetti et al. 2013; Shaw et al. 2013a,b). Therefore additional observations are needed in order to obtain a significant sample that allows a detailed statistical analysis to firmly assess the completeness and efficiency of our selection methods.

\section{References}

Ackermann, M., et al., 2011, ApJ, 743, 171

Aharonian, F., et al., 2009, A\&\&A, 502, 749

Berlin, A. \& Hooper, D. 2014, Phys. Rev. D., 89, 016014

Blandford, R. D. \& Rees, M. J., 1978, Proc. "Pittsburgh Conference on BL Lac objects," 328

Cowperthwaite, P. S., Massaro, F., D'Abrusco, R., et al. 2013, AJ, 146, 110

D'Abrusco, R., Longo, G., \& Walton, N. A., 2009, MNRAS, 396, 223

D'Abrusco R., Massaro F., Ajello M., Grindlay J. E., Smith H. A., Tosti G., 2012, ApJ, 748, 68

D'Abrusco, R., Massaro, F., Paggi, A., et al., 2013, ApJS, 206, 12

Dermer, C. D. \& Schlickeiser, R., 1993, ApJ, 416, 458

Dermer, C. D., Finke, J. D., Krug, H., \& Böttcher, M., 2009, ApJ, 692, 32

Giommi, P., Padovani, P., \& Polenta, G., 2013, MNRAS, 431, 1914

Inoue, S. \& Takahara, F., 1996, ApJ, 463, 555

Landoni, M., Falomo, R., Treves, A., et al. 2012, A\& A, 543, A116

Landoni, M., Falomo, R., Treves, A., et al. 2013, AJ, 145, 114

Landoni, M., Massaro, F., Paggi, A., et al. 2014, AJ, submitted

Laurent-Muehleisen, S. A., Kollgaard, R. I., Feigelson, E. D., et al. 1999, ApJ, 525, 127

Laurino, O., D’Abrusco, R., Longo, G., \& Riccio, G., 2011, MNRAS, 418, 2165

Londish, D., Heidt, J., Boyle, B. J., et al. 2004, MNRAS, 352, 903

Masetti, N., Sbarufatti, B., Parisi, P. 2013 A $\& A$, 559, A58

Massaro, E., Giommi, P., Leto, C., et al. 2013, VizieR Online Data Catalog, 349, 50691

Massaro, F., D'Abrusco, R., \& Ajello, M., Grindlay J. E., Smith H. A., 2011, ApJ, 740, L48

Massaro, F., D'Abrusco, R., Paggi, A., et al., 2013a, ApJS, 206, 13

Massaro, F., D'Abrusco, R., Paggi, A., et al. 2013b, ApJS, 209, 10

Massaro, F., D'Abrusco, R., Giroletti, M., et al. 2013c, ApJS, 207, 4

Massaro, F., Landoni, M., D'Abrusco, R., et al. 2014, AJ, submitted

Nolan P. L., et al., 2012, ApJS, 199, 31

Paggi, A., Massaro, F., D'Abrusco, R., et al. 2013, ApJS, 209, 9

Paggi, A., Milisavljevic, D., Masetti, N., et al. 2014, AJ, 147, 112

Ricci, F., Massaro, F., Landoni, N., et al. 2014, in preparation

Richards, G. T., et al., 2004, ApJS, 155, 257

Sbarufatti, B., Treves, A., Falomo, R., et al. 2006, AJ, 132, 1

Sbarufatti, B., Ciprini, S., Kotilainen, J., et al. 2009, AJ, 137, 337

Shaw, M. S., et al., 2013a, ApJ, 764, 135

Shaw, M. S., Filippenko, A. V., Romani, R. W. et al. 2013b AJ, 146, 127

Shemmer, O., et al., 2006, ApJ, 644, 86

Shemmer, O., Brandt W. N., Anderson S. F., et al., 2009, ApJ, 696, 580

Stern, D. \& Assef, R. J. 2013 ApJ, 764, L30

Stickel, M., Padovani, P., Urry, C. M., Fried, J. W., \& Kuehr, H., 1991, ApJ, 374, 431

Stocke J. T. \& Rector T. A., 1997, ApJ, 489, L17

Urry C. M. \& Padovani P., 1995, PASP, 107, 803 